\title{
Indices for the assessment of nutritional quality of meals: a systematic review
}

\author{
B. M. Gorgulho ${ }^{1}$, G. K. Pot $^{2}$, F. M. Sarti ${ }^{1,3}$ and D. M. Marchioni ${ }^{1 *}$ \\ ${ }^{1}$ Department of Nutrition, School of Public Health, University of São Paulo, Avenida Dr. Arnaldo 715, CEP 01246-904, São \\ Paulo, SP, Brazil \\ ${ }^{2}$ Diabetes and Nutritional Sciences Division, 2.8N Hodgkin Building, Guy's Campus, King's College London, London SE1 1UL, UK \\ ${ }^{3}$ School of Arts, Sciences and Humanities, University of São Paulo, Rua Arlindo Bettio 1000, CEP O3828-000, São Paulo, SP, Brazil \\ (Submitted 4 August 2015 - Final revision received 13 February 2016 - Accepted 18 February 2016 - First published online 5 April 2016)
}

\section{Abstract}

This systematic review aimed to synthesise information on indices developed to evaluate nutritional quality of meals. A strategy for systematic search of the literature was developed using keywords related to assessment of meal quality. Databases searched included ScienceDirect, PubMed, Lilacs, SciELO, Scopus, Cochrane, Embase and Google Scholar. The literature search resulted in seven different meal quality indices. Each article was analysed in order to identify the following items: authors, country, year, study design, population characteristics, type of meal evaluated, dietary assessment method, characteristics evaluated (nutrients or food items), score range, index components, nutritional references, correlations performed, validation and relationship with an outcome (if existing). Two studies developed instruments to assess the quality of breakfast, three analysed lunch, one evaluated dinner and one was applied to all types of meals and snacks. All meal quality indices reviewed were based on the evaluation of presence or absence of food groups and relative contributions of nutrients, according to food-based guidelines or nutrient references, adapting the daily dietary recommendations to one specific meal. Most of the indices included three items as components for meal quality assessment: (I) total fat or some specific type of fat, (II) fruits and vegetables and (III) cereals or whole grains. This systematic review indicates aspects that need further research, particularly the numerous approaches to assessing meals considering different foods and nutrients, and the need for validation studies of meal indices.

\section{Key words: Meal quality: Meal index: Meal score: Nutrition: Systematic reviews}

The influence of food intake on health is unquestionable ${ }^{(1)}$. However, because of the complexity of diet and the potential for interactions among dietary components, approaches that focus on individual foods or nutrients may miss information on the role of diet in disease aetiology. In this way, a more holistic approach has been applied, and, since the 1990, dietary indices to evaluate the global quality of meals and diets have been proposed ${ }^{(2,3)}$. These instruments are pre-defined summary measures of overall food intake that may be used as a simple and quick instrument for the assessment of nutritional quality, in order to evaluate adherence to dietary guidelines and to monitor overall changes in food consumption patterns ${ }^{(4)}$. Nonetheless, although the idea of analysing food intake at the meal level is not exactly new ${ }^{(5-8)}$, data on the nutritional composition of meals are scarce in the literature.

Meals are eating events that include certain combinations of foods and beverages in a concentrated period, combining taste and food consumption. Definitions of meal have been described from a number of different perspectives such as self-report by participants, time of the day and energy content ${ }^{(9,10)}$. Identifying and analysing meals allow us to understand how different combinations of foods and beverages at eating events could influence the overall diet quality and health outcomes ${ }^{(11-13)}$

Nutritional advices considering a meals-based approach might be easier and more practical for the population to understand and follow dietary guidelines ${ }^{(14)}$. In 2014, the Brazilian Ministry of
Health published the first food guide with recommendations for meals, highlighting the commensality importance of healthy food habits ${ }^{(15)}$. The new guide also recommends moderate use of food products that are ready for consumption and encourages the consumption of fresher foods such as fruits and vegetables ${ }^{(15)}$.

There are some systematic reviews performed to date on healthy eating indices and other indicators to evaluate global dietary quality ${ }^{(2-4,16,17)}$. However, thus far, no systematic review has described and compared instruments developed to summarise meals, measure meals' nutritional quality and evaluate the adherence of meals to the nutritional recommendations. Considering the Cochrane recommendations, Preferred Reporting Items for Systematic Reviews and Meta-Analyses (PRISMA) checklist and Population Intervention Comparison Outcome (PICOS) strategy, the aim of this systematic review was to describe and compare indices (pre-defined in the literature) for the assessment of nutritional quality of meals.

\section{Methods}

\section{Search strategy, selection criteria and data extraction}

A literature search for original studies on meal quality index was performed in order to achieve the results presented in this systematic review. Databases searched included the 
Table 1. Inclusion and exclusion criteria used for the present review

\begin{tabular}{lll}
\hline Study characteristics & Inclusion criteria & Exclusion criteria \\
\hline $\begin{array}{l}\text { Population } \\
\text { Intervention }\end{array}$ & $\begin{array}{c}\text { Studies with humans } \\
\text { Evaluation of meal quality using a composite index } \\
\text { proposed to synthesise nutritional quality } \\
\text { Methodological instrument to assess of overall } \\
\text { nutritional quality of meals } \\
\text { Observational studies, cohort studies, case-control, } \\
\text { systematic review, meta-analysis }\end{array}$ & $\begin{array}{l}\text { Studies with no humans } \\
\text { Description of meal quality according to content of nutrients }\end{array}$ \\
Study type & $\begin{array}{c}\text { Analysis without results on development, evaluation or application } \\
\text { of a composite index to synthesise nutritional quality } \\
\text { Animal research }\end{array}$ \\
\hline
\end{tabular}

following: ScienceDirect, PubMed, Lilacs, SciELO, Scopus, Cochrane, Embase and Google Scholar. A search strategy was developed using keywords related to the assessment of nutritional quality of meals and was initially developed in PubMed and adapted for use in other databases. The following search string was used: (meal* OR lunch OR dinner OR breakfast OR meal quality OR dietary pattern OR dietary quality OR eating index OR restaurant menu OR menu component OR meal score OR meal plate OR food service). The search was limited by language (English and Portuguese) and year of publication (1965 until 2014). Using an adaptation of the PICOS strategy ${ }^{(18)}$, inclusion and exclusion criteria are described in Table 1.

Articles that presented results on the development, evaluation and application of methodological instruments aimed to assess the nutritional quality of meals were considered eligible for inclusion, regardless of the definition used to classify the eating events (self-report, period of consumption, energy content or neutral). In addition, there were no restrictions in relation to the dietary assessment methods used or potential outcomes associated with the meal quality. Duplicate articles were identified and eliminated using key terms such as first author, publication year, journal title, volume and number of the first page. The title and abstract of relevant articles and potentially relevant references were analysed, and references and bibliographies were hand searched in order to provide further contributions to the systematic review. The assessment of articles and data extraction were carried out by two independent reviewers (B. M. G. and D. M. M.), applying the inclusion criteria described. Discrepancies in relation to the papers and data to be included in the systematic review were solved by a third reviewer.

A database containing detailed information from each article was developed, in order to allow a comprehensive analysis of the meal quality indices proposed: authors, country, year, study design, population characteristics, type of meal evaluated, dietary assessment method, characteristics evaluated (nutrients or food items), score range, index components, nutritional references, correlations performed, validation and relationship with an outcome (if existing).

A narrative review of the seven studies included was performed, the studies were compared and the key issues to be considered to develop an indicator were discussed, including the components, cut-off values and scoring ${ }^{(3)}$. Data on content and construct validity and reliability of the indicators were observed. Guenther et al. (19) suggested that besides the correlation between the scores and nutrients and guidelines, it is necessary to verify whether the indicator can distinguish groups with known differences in diet quality, detect more than one dimension of intake and be sensitive to identify meaningful differences.

The systematic review protocol was developed according to the Cochrane recommendations ${ }^{(20)}$ and the PRISMA ${ }^{(21)}$. The systematic review protocol was registered at the international prospective register of systematic reviews, PROSPERO ${ }^{(22)}$ (registration no. CRD42014013519).

\section{Results}

\section{Studies included in the systematic review}

The flow diagram of the systematic search is presented in Fig. 1. The literature search resulted in 22963 citations. The initial screening of the references based on title excluded 21038 references, and 548 duplicate articles were identified and removed. The second screening of the references based on the contents of the abstract excluded 730 references. Five new studies were identified after examining the list of references from the selected papers and these were included in the review. The main reason for exclusion was that most of the studies did not evaluate the nutritional quality of the meals using a composite index as an instrument to synthesise the measurement of nutritional quality, such as a score or a checklist.

Finally, eight studies were identified. Among these, two were about the same instrument ${ }^{(23,24)}$, resulting in seven indices included in this systematic review (Table 2). With respect to the country of origin, two studies were carried out in Spain ${ }^{(25,26)}$, two in Denmark ${ }^{(27,28)}$, one in Sweden ${ }^{(29)}$, one in the USA ${ }^{(30)}$ and one in Brazil ${ }^{(23)}$. Among the studies included in the systematic review, none of them investigated the meal quality relationship with health outcomes.

In relation to nutritional intake, three of the selected studies analysed the meal quality considering a qualitative description $^{(25,29,30)}$, whereas three studies adopted a quantitative $\operatorname{approach}^{(23,27,28)}$, and one used both possibilities ${ }^{(26)}$ (Table 2).

\section{Type of meal evaluated}

Most researchers have defined meals and snacks separately, classifying the eating event based on participant/institution report $^{(23,27,28)}$ and period of day ${ }^{(26,30)}$. Only one study used a neutral definition, considering both meals and snacks ${ }^{(29)}$. Two studies developed an instrument for the assessment of breakfast quality $^{(25,26)}$, three analysed lunch ${ }^{(23,27,28)}$, one evaluated dinner $^{(30)}$ and one was applied to all types of meals and 


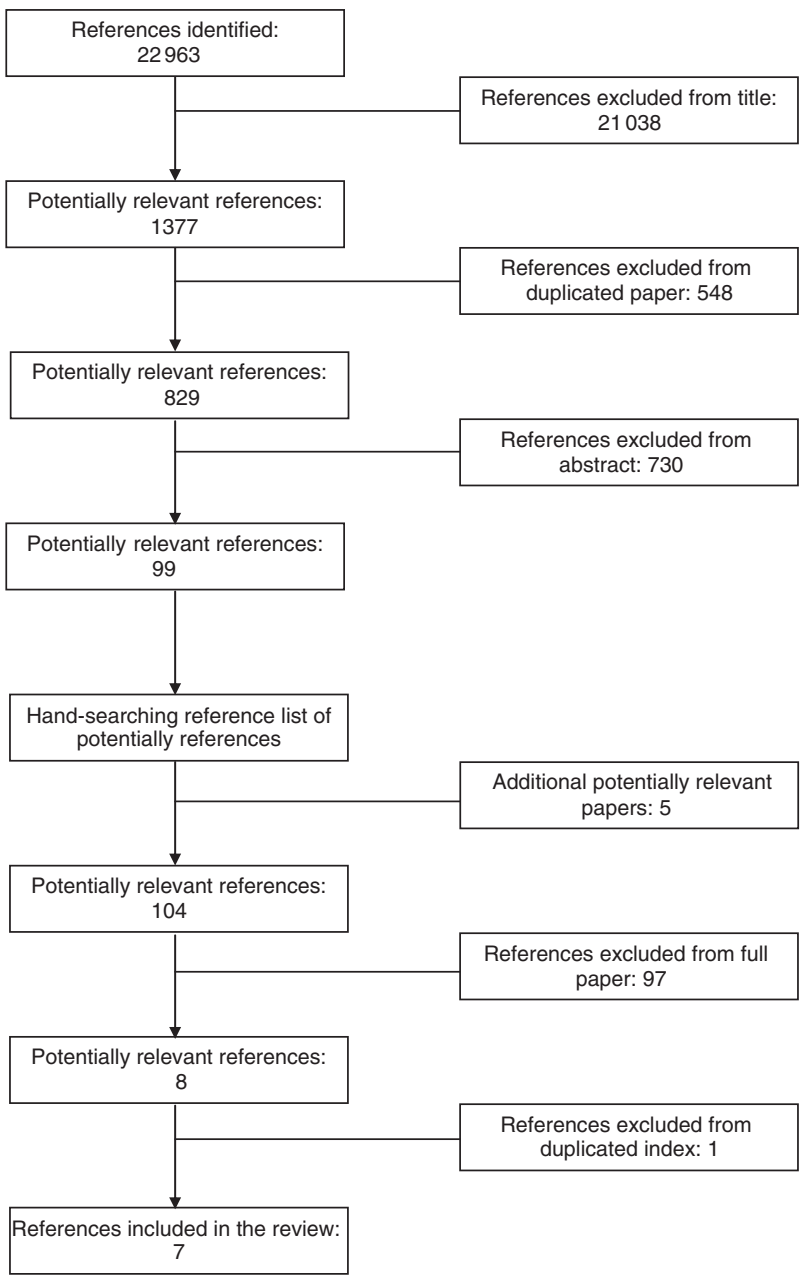

Fig. 1. Flow diagram illustrating the screening process of eligible studies.

snacks ${ }^{(29)}$. The type of meal evaluated in each study is presented in Table 2.

The Breakfast Quality Index ${ }^{(26)}$ and the Breakfast Score ${ }^{(25)}$ were specifically developed to evaluate the nutritional quality of breakfasts consumed by children and adolescents, and should not be used to evaluate other types of meals. The components of these indices were based on foods typically consumed at breakfast, excluding other food groups.

The Meal Index of Dietary Quality ${ }^{(28)}$, the Meal Quality Index ${ }^{(23)}$ and the Healthy Meal Quality ${ }^{(27)}$ were developed to evaluate the quality of lunch, and the index Five Food Group and Healthfulness Score ${ }^{(30)}$ was developed to evaluate the quality of dinner. However, considering the components proposed, the four indices could be applied for lunch and dinner. In fact, the difference is that the Meal Index of Dietary Quality was proposed for meals consumed by adolescents at school, the Meal Quality Index and the Healthy Meal Quality for meals consumed by adults at work and the Five Food Group and Healthfulness Score for meals served at home by adults residing with the child.

The meal classifications system was used to evaluate all types of meals and snacks consumed by elderly women, reflecting eating frequency and key nutrients ${ }^{(29)}$. 


\section{Dietary assessment method}

The dietary assessment method used in each study is presented in Table 2. The studies that were analysed in the systematic review were based on the collection of dietary information through the application of $24-\mathrm{h}$ recalls ${ }^{(25)}$, food diaries ${ }^{(29)}, \mathrm{FFQ}^{(26)}$, menus $^{(23)}$, weighted foods ${ }^{(27,28)}$ and meal screeners ${ }^{(30)}$. The methods observed for data collection regarding dietary intake are the ones traditionally used for research in nutrition, with exception of the meal screener, which was proposed and validated especially for the development of the meal quality index in the study conducted by Fulkerson et al. ${ }^{(30)}$. The meal screener is an open-ended questionnaire for participants to describe foods that were served and consumed, including specific questions about the type of food and the method of preparation ${ }^{(30)}$

\section{Nutritional references}

The nutritional references used to develop the indices included in this review were as follows: the Food Guide Pyramid developed by the US Department of Agriculture (USDA) ${ }^{(31)}$, the Danish Dietary Guidelines ${ }^{(32)}$, the Mediterranean Diet Guidelines $^{(33)}$, the Brazilian Dietary Guidelines ${ }^{(34)}$, the Swedish Nutrition Recommendations ${ }^{(35)}$ and the Energy and Nutrients Recommendations for the Spanish Population ${ }^{(36)}$. The Food Guide Pyramid and the Danish, Mediterranean and Brazilian guidelines were based on food items, meaning that they usually are based on suggestions regarding intake of food portions and portion sizes, whereas the Swedish and Spanish nutritional recommendations are based on quantitative intake, in grams or percentage contribution of energy and nutrients ${ }^{(37)}$.

The nutritional references suggest dietary recommendations for daily food intake, considering the combination of meals and snacks consumed during $1 \mathrm{~d}$. Therefore, these indices were based on the evaluation of presence or absence of food groups and relative contributions of nutrients recommended, adapting the recommendations for a dietary day to one specific meal.

\section{Components}

The components of each indicator included in the systematic review are presented in Table 3. The number of components among the indices ranged between three and fifteen items. Most of the indices included three items as components for meal quality assessment: (I) total fat or some specific type of fat $^{(23,26-29)}$, (II) fruits and vegetables ${ }^{(23,26-29)}$ and (III) cereals or whole grains ${ }^{(25-28)}$. Other items usually prevalent in meal quality indices were dairy products and $\mathrm{Ca}^{(25,26)}$, diversity of food items and food groups ${ }^{(23,30)}$ and added sugar ${ }^{(28,29)}$.

The Five Food Group and Healthfulness Score, the Breakfast Score and the Qualitative Meal Classifications System were based on the evaluation of meal quality using food items and food groups as components ${ }^{(25,29,30)}$. The Meal Quality Index, the Healthy Meal Quality, the Breakfast Quality Index and the Meal Index of Dietary Quality were based on the assessment of meal quality using food items, food groups and nutrients as components $^{(23,26-28)}$. None of the studies proposed the evaluation of the nutritional quality of meals based on indices that included only nutrients (Table 3).

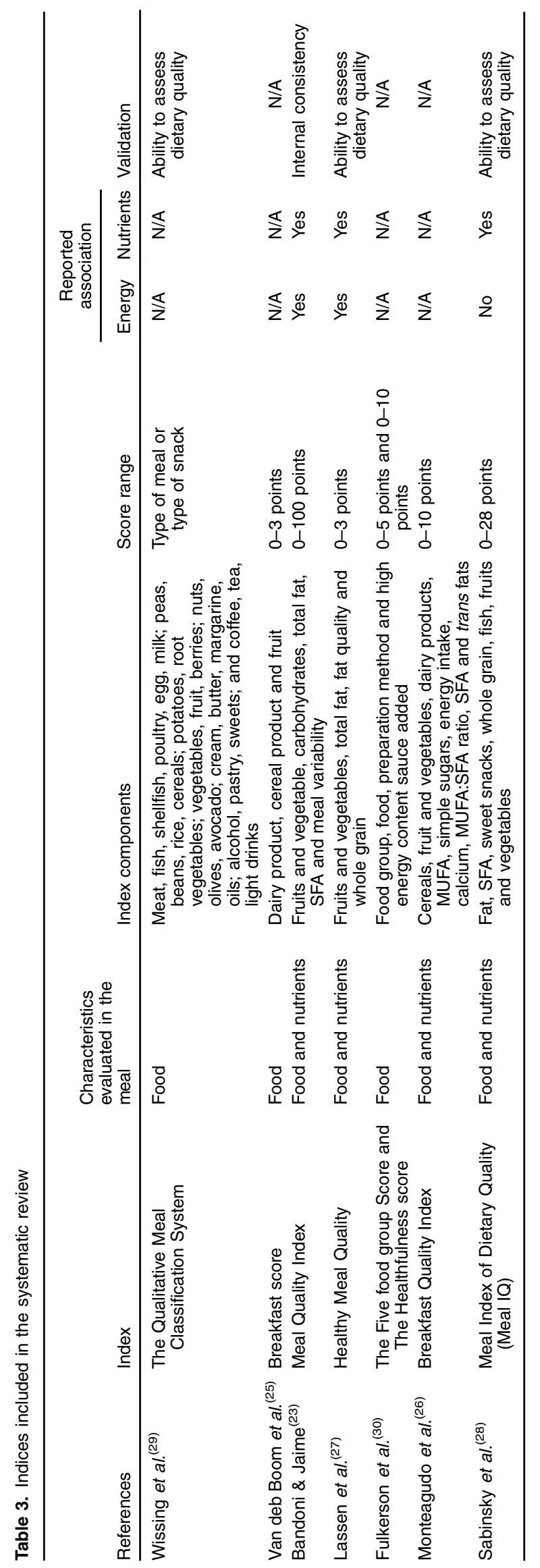


The Five Food Group and Healthfulness Score was based on the composition of two scale scores: the Five Food Group Score, in order to assess the presence of food items from the five major groups of the Food Guide Pyramid developed by the USDA, and the Healthfulness Scale Score, in order to assess the healthfulness of foods based on preparation methods and added fats. The Five Food Group Score assigns one point for the inclusion of at least one food item from each food group (range $=0-5$ ), and the Healthfulness Scale Score assigns one point for the inclusion of food items from each major group plus one point for the adoption of healthy preparation methods and subtracts one point for the inclusion of sauces with high energy content (range $=0-10)^{(30)}$

The Breakfast Score considered the consumption of three groups (dairy products, cereals and fruits), scoring one point for each group consumed during breakfast. Thus, a complete breakfast that included a dairy product, a cereal and a fruit should obtain a maximum of 3 points ${ }^{(25)}$.

The Qualitative Meal Classification System was based on eight food categories (representing one key nutrient each) combined into four types of meal (complete, incomplete, less balanced and vegetarian meals) and three types of snacks (high-, mixed- and low-quality snacks) ${ }^{(29)}$.

The Meal Quality Index was composed of five components namely, content of fruits and vegetables, content of carbohydrates, content of total fat, content of SFA and menu variety - scoring each component from 0 to 20 points (range $=0-100)^{(23)}$

The Healthy Meal Quality and the Meal Index of Dietary Quality came from the same research group, and, although very similar, were developed for different populations ${ }^{(27,28)}$. The Healthy Meal Index was developed to reflect the nutritional profile of canteen meals ${ }^{(27)}$, whereas the Meal Index of Dietary Quality was developed to assess the dietary quality of school lunches ${ }^{(28)}$. Three components were prioritised in the Healthy Meal Index: fruits and vegetables, total fat and fat quality, and whole grains and potatoes. Each component could be scored from 0 to 1 point and were added up to determine the overall score (range $=0-3)^{(27)}$. The Meal Index of Dietary Quality considered seven components including nutrients and food groups - fat, SFA, sweet snacks, whole grain, fish, fruits and vegetables - scoring from 0 to 4 for each component (range $=0-28)^{(28)}$

The Breakfast Quality Index, similarly to the Breakfast Score, took into account the consumption of dairy products, cereals and fruits, also considering the content of selected nutrients. The Breakfast Quality Index scores 1 point for the consumption of each of the following food items and nutrients: dairy products, cereals, fruit/vegetables, MUFA, sugar (up to $5 \%$ of total daily energy consumption), energy intake (between 20 and $25 \%$ of total daily energy intake), Ca (between 200 and $300 \mathrm{mg}$ ), MUFA:SFA ratio above the population median, combination of items from cereals, dairy products and fruit/ vegetables groups in one meal and non-consumption of foods with high content of SFA or trans fatty acids (range $=1-10)^{(26)}$.

\section{Cut-off points of the indices and their components}

The cut-off point of each index and its components are presented in Table 3. Six of the studies included in the systematic review proposed the calculation of a score for the nutritional index ${ }^{(23,25-28,30)}$. Indices based on qualitative assessment approach included components on the consumption of specific food groups, attributing a score of 0 if the food group was not consumed and 1 if the food group was consumed ${ }^{(25,26,30)}$. The Meal Index of Dietary Quality and the Meal Quality Index proposed cut-off values for each component, attributing a score of 0 if consumption was lower/higher than this value for items considered healthy/unhealthy (respectively), a score of 1 if consumption was higher/lower than the cut-off for items considered healthy/unhealthy (respectively) and intermediate values of consumption receiving proportional scores ${ }^{(23,28)}$.

None of the studies proposed weighting of the items included in the calculation of the index, presenting the components with the same relative importance in the score ${ }^{(23,25-28,30)}$. The Healthy Meal Quality ${ }^{(27)}$ and the Breakfast Score ${ }^{(25)}$ presented the lower score ranges (three points), and the Meal Quality Index (100 points) presented the highest score range ${ }^{(23)}$.

\section{Validation and correlations with energy and nutrients}

The validity and correlations with nutrients verified by each indicator included in the systematic review are presented in Table 3. Certainly, the main risk of bias for these indicators is the insufficiency (or absence) of validity and reliability analysis described in the published studies.

Four studies showed some validity analysis of the meal quality indices presented ${ }^{(23,27-29)}$. Among these, three studies evaluated the ability of the index to assess dietary quality using one-way ANOVA followed by Tukey's post hoc test, if normally distributed, or the Kruskal-Wallis test followed by the Mann-Whitney post hoc test, assessing differences between the categories ${ }^{(27-29)}$. However, the internal consistency of the components was evaluated only in one study, which applied the Cronbach's $\alpha^{(23)}$. Furthermore, using Pearson's and Spearman's correlation coefficients, the studies that proposed quantitative assessments had analysed the linear relationships between the scores of each component of the indices in relation to the contents of energy and nutrients in the meals ${ }^{(23,27,28)}$.

For the Healthy Meal Index, no significant differences were found with respect to the tested background variables (i.e. age, sex and BMI) over the categories of classification. Energy density, total fat, SFA, carbohydrate, and fruits and vegetables were highly, significantly associated, with higher scores being closer to dietary guidelines ${ }^{(27)}$. The ANOVA showed significant differences between the Meal Index of Dietary Quality scores. A higher score was associated with lower intakes of total fat and SFA and sugar and with higher intakes of fibre, fish, fruits and vegetables $^{(28)}$. The Meal Quality Index had a Cronbach's $\alpha$ value higher than $0 \cdot 60^{(23)}$, as is recommended, and the qualitative meal classification method found significant differences between the meal classifications - completed meals contained more $\beta$-carotene and ascorbic acid ${ }^{(29)}$.

\section{Discussion}

Although there is widespread recognition of the importance of nutrition and diet for the prevention of chronic diseases, there 
are only a few studies investigating the dietary characteristics of meals. This is the first systematic review on indices proposed to evaluate the nutritional quality of meals. Overall, we found seven indices developed to evaluate the quality of breakfast, lunch and dinner.

None of the articles presented an analysis of the relationship between meal quality and health outcomes; however, the indices were developed based on nutritional recommendations designed to prevent overweight ${ }^{(36)}$ and, consequently, to target the increasing prevalence of chronic diseases. The evaluation of the nutritional quality of a single meal will not necessarily reflect the daily dietary quality, restricting the direct association between indices and health outcomes. The association of the meal quality with overall dietary intake could be valuable to observe the associations between each meal and dietary quality.

Despite cultural differences, the recommendations for a healthy diet for most countries ${ }^{(36)}$ and for international agencies $^{(37)}$ consistently are high consumption of whole grains, fruits and vegetables, associated with the reduction of total fat and SFA and added sugar. The main purpose of the indices found in the systematic review was to evaluate the adherence of individuals to nutritional recommendations, proposing specific indicators for each country and each population group under assessment. Although indices designed for the assessment of each meal seemed to be developed with this view, the results cannot be easily compared across studies, considering their different components, score ranges and populations targeted such as students and workers. Moreover, there is no consensus in the literature regarding the definition of eating occasions ${ }^{(10)}$. Some authors define meals considering the consumption time slot or nutrient content, whereas other authors define it based on the respondent report ${ }^{(10)}$. This difference in the definitions makes more difficult the comparison of findings; a recent study showed that, depending of the meal definition used, meal frequency can be, or not, associated with nutritional status ${ }^{(38)}$.

Composing a nutritional index is complex and involves many choices related to components, including cut-off values to compare with reported intake, and scoring methods ${ }^{(14,19)}$. The assessment of nutritional quality of meals considering nutrients and food groups is usually a strategy used to facilitate the comparison of the dietary quality among different populations. Although there are new proposals for dietary guidelines based on nutritional recommendations for meals ${ }^{(12)}$, there is still lack of quantitative dietary recommendations regarding the meal quality.

The seven meal quality indices identified in this review were based on the assumption that all components have the same impact on health. However, it is possible that some components may present higher weight than others in determining populations' and individuals' health outcomes. Two indices cited were based on the evaluation of presence or absence of certain food items or food groups, and did not consider the amount of each food served and/or consumed ${ }^{(25,30)}$. Nevertheless, it is preferable that the score range represents a proportional measure in relation to energy intake, instead of proposing simple cut-off values, as larger score ranges allow a better distribution of the population studied, identifying possible intake differences between individuals.
The process for development of a nutritional indicator includes its validation in order to evaluate whether the indicator captures the targeted aspects of the nutritional references, its construct validity and reliability ${ }^{(19)}$. A suitable nutritional indicator may be able to distinguish between consumption groups with significant differences in nutritional quality and to recognise the influence of each component on the overall score of the index ${ }^{(13)}$. Although internal validity analysis is considered essential to test the indices' ability to properly measure the dimensions under evaluation ${ }^{(39)}$, not all studies presented the test for the proposed indices and their components.

A statistical strategy commonly used to verify the properties of the composite indices proposed for assessment of nutritional quality of meals was the correlation analysis between the score of meal quality index and the amount of macronutrients and/or micronutrients reported in dietary questionnaires ${ }^{(25,26)}$. In addition, it is important to remember that the final score of the indices should be adjusted for energy intake in order to avoid confounding factors ${ }^{(19,40)}$.

\section{Limitations}

One of the limitations of this study was the inclusion of articles published mainly in English. As the indices were specific for the country's dietary recommendations, it is possible that there are other publications on the assessment of the nutritional quality of meals in other languages.

Despite all concerns during the literature review and the extensive search in different databases with different combinations of search terms, it is possible that some manuscripts that comprise inclusion criteria have not been found or not published until the conclusion of this systematic review. Furthermore, this review was limited to composite index and does not include other methods to assess the quality of meals.

\section{Conclusion}

The development and application of meal quality indices are relatively new, beginning to be used more often since the last decade. It is noteworthy that the meal quality indices found during the systematic review still need to be internally validated, as it is important to know whether the index reflects the most important nutritional aspects of the dietary intake reported and, in fact, whether it assesses the adherence to nutritional recommendations. Moreover, it is important to propose nutritional indices tailored to specific purposes in public health, because of their importance in the evaluation of determinants of populations' health and their role as tools for promoting longterm quality of life.

Mostly, the dietary guidelines used as foundations to develop meal quality indices were quite similar among the different countries. There are aspects that still warrant further research, particularly the numerous approaches to assessing meals considering different foods and nutrients, and the need for validation studies of the indices designed for the assessment of the nutritional quality of meals. Nevertheless, the possibility to have a single indicator developed for the assessment of meal quality, designed to allow the comparison of population-level food intake in different countries, should be useful for public 
health. The comparative analysis of countries at different epidemiological and nutritional stages could bring advances in the development of public policies for the promotion of population health based on food and nutrition initiatives.

\section{Acknowledgements}

The authors thank Petra Wark for her contribution to the earlier stages, that is, for the definition of the research question and initial scoping of the project.

This study was funded by the Brazilian Council for Scientific and Technological Development (process number 142341/ 2013-4) and by the São Paulo Research Foundation (process number 2014/19355-6).

All the authors contributed towards the study's concepts and design and towards data analysis and interpretation.

The authors declare that there are no conflicts of interest.

\section{References}

1. World Health Organization (2003) Diet, Nutrition and the Prevention of Chronic Diseases. WHO Technical Report Series no. 916, pp. 1-60. Geneva: WHO.

2. Ocke MC (2013) Evaluation of methodologies for assessing the overall diet: dietary quality scores and dietary pattern analysis. Proc Nutr Soc 72, 191-199.

3. Waijers PM, Feskens EJ \& Ocké MC (2007) A critical review of predefined diet quality scores. Br J Nutr 97, 219-231.

4. Fransen HP \& Ocke MC (2008) Indices of diet quality. Curr Opin Clin Nutr Metab Care 11, 559-565.

5. Hedderley DI \& Meiselman HL (1995) Modelling meal acceptability in a free choice environment. Food Qual Prefer 6, 15-26.

6. Oltersdorf U, Schlettwein-Gsell D \& Winkler G (1999) Assessing eating patterns-an emerging research topic in nutritional sciences: introduction to the symposium. Appetite 32, 1-7.

7. Rozin P (2005) The meaning of food in our lives: a cross-cultural perspective on eating and well-being. $J$ Nutr Educ Behav 37, S107-S112.

8. Hearty AP \& Gibney MJ (2008) Analysis of meal patterns with the use of supervised data mining techniques - artificial neural networks and decision trees. Am J Clin Nutr 88, 1632-1642.

9. Leech RM, Worsley A, Timperio A, et al. (2015) Characterizing eating patterns: a comparison of eating occasion definitions. Am J Clin Nutr 102, 1229-1237.

10. Meiselman HL (2008) Dimensions of the meal. J Foodserv 19, $13-21$.

11. Costa AIdA, Schoolmeester D, Dekker M, et al. (2007) To cook or not to cook: a means-end study of motives for choice of meal solutions. Food Qual Prefer 18, 77-88.

12. Fjellström K (2008) Mealtime and meal patterns from a cultural perspective. Food Nutr Res 48, 161-164.

13. Popkin BM \& Duffey KJ (2010) Does hunger and satiety drive eating anymore? Increasing eating occasions and decreasing time between eating occasions in the United States. Am J Clin Nutr 91, 1342-1347.

14. Leech RM, Worsley A, Timperio A, et al. (2015) Understanding meal patterns: definitions, methodology and impact on nutrient intake and diet quality. Nutr Res Rev 28, 1-21.

15. Brasil. Ministério da Saúde. Secretaria de Atenção à Saúde. Departamento de Atenção Básica (2014) Guia Alimentar Para a População Brasileira (Dietary Guidelines for the Brazilian Population). Brasília: Ministério da Saúde.
16. Ruel MT (2003) Operationalizing dietary diversity: a review of measurement issues and research priorities. J Nutr 133, Suppl. 2, 3911s-3926s

17. Kourlaba G \& Panagiotakos DB (2009) Dietary quality indices and human health: a review. Maturitas 62, 1-8.

18. Santos CMdC, Pimenta CAdM \& Nobre MRC (2007) The PICO strategy for the research question construction and evidence search. Rev Lat Am Enfermagem 15, 508-511.

19. Guenther PM., Reedy J, Krebs-Smith SM, et al. (2008) Evaluation of the healthy eating index-2005. J Am Diet Assoc 108, 1854-1864.

20. Higgins JP \& Green S (2008) Cochrane Handbook for Systematic Reviews of Interventions, vol. 5. Chichester: Wiley Online Library.

21. Moher D, Liberati A, Tetzlaff J, et al. (2009) Preferred reporting items for systematic reviews and meta-analyses: the PRISMA statement. Ann Intern Med 151, 264-269.

22. Chien PF, Khan KS \& Siassakos D (2012) Registration of systematic reviews: PROSPERO. BJOG 119, 903-905.

23. Bandoni DH \& Jaime PC (2008) The quality of meals in companies participating in the workers food program in the city of São Paulo, Brazil. Rev Nutr 21, 177-184.

24. Sampaio Moreira Barbosa R, Serrão Lanzillotti H \& Henriques P (2014) Reproducibility study of the meal quality index. Rev Chilena Nutr 41, 72-76.

25. van den Boom A, Serra-Majem L, Fau-Ribas L, et al. (2006) The contribution of ready-to-eat cereals to daily nutrient intake and breakfast quality in a Mediterranean setting. J Am Coll Nutr 25, 135-143.

26. Monteagudo C, Palacin-Arce A, Bibiloni Mdel M, et al. (2013) Proposal for a Breakfast Quality Index (BQI) for children and adolescents. Public Health Nutr 16, 639-644.

27. Lassen AD, Biltoft-Jensen A, Hansen GL, et al. (2010) Development and validation of a new simple healthy meal index for canteen meals. Public Health Nutr 13, 1559-1565.

28. Sabinsky MS, Toft U, Andersen KK, et al. (2012) Development and validation of a Meal Index of dietary Quality (Meal IQ) to assess the dietary quality of school lunches. Public Health Nutr 15, 2091-2099.

29. Wissing U, Lennernas MAC, Ek AC, et al. (1998) Monitoring of dietary quality in outpatients by qualitative meal classification method. J Human Nutr Diet 11, 125-133.

30. Fulkerson JA, Lytle L, Story M, et al. (2012) Development and validation of a screening instrument to assess the types and quality of foods served at home meals. Int J Behav Nutr Phys Act 9, 1-7.

31. Shaw AM, Fulton L, Davis C, et al. (2001) Using the Food Guide Pyramid: A Resource for Nutrition Educators. Oklahoma City, OK: Oklahoma State Department of Education.

32. Mithril C, Dragsted LO, Meyer C, et al. (2013) Dietary composition and nutrient content of the new Nordic diet. Public Health Nutr 16, 777-785.

33. Martnez E, Llull R, Del Mar Bibiloni M, et al. (2010) Adherence to the mediterranean dietary pattern among Balearic islands adolescents. Br J Nutr 103, 1657-1664.

34. Brasil. Ministério da Saúde. Secretaria de Atenção à Saúde. Departamento de Atenção Básica (2006) Guia Alimentar Para a População Brasileira: Promovendo a Alimentação Saudável (Dietary Guidelines for the Brazilian Population: Promoting Healthy Food). Brasília: Ministério da Saúde.

35. Barbieri HE, Lindvall C \& Livsmedelsverket S (2005) Swedish Nutrition Recommendations Objectified (SNO): Basis for General Advice on Food Consumption for Healthy Adults. Uppsala: Livsmedelsverket.

36. Navia B \& Ortega R (2000) Ingestas recomendadas de energía y nutrientes (Recommended energy and nutrient intakes). In Nutriguía: Manual de Nutrición Clínica en Atención Primaria 
(Nutriguia: Manual of Clinical Nutrition in Primary Care), pp. 3-13 [AM Requejo and RM Ortega, editors]. Madrid: Editorial Complutense.

37. World Health Organization (1990) Diet, Nutrition and the Prevention of Chronic Diseases. Geneva: WHO.

38. Murakami K \& Livingstone MB (2015) Associations between meal and snack frequency and diet quality and adiposity measures in British adults: findings from the
National Diet and Nutrition Survey. Public Health Nutr 16 , $1-11$.

39. Nardo M, Saisana M, Saltelli A, et al. (2005) Handbook on Constructing Composite Indicators: Methodology and User Guide. Paris: OECD Publishing.

40. Jaime PC, Bandoni DH, Duran ACFL, et al. (2010) Diet quality index adjusted for energy requirements in adults. Cad Saúde Pública 26, 2121-2128. 